\title{
Proximity bounds for random integer programs
}

\author{
Marcel Celaya ${ }^{\star}$ and Martin Henk \\ Technische Universität Berlin \\ Institut für Mathematik, Sekr. MA4-1 \\ Straße des 17 Juni 136 \\ D-10623 Berlin \\ \{henk,celaya\}@math.tu-berlin.de
}

No Institute Given

\begin{abstract}
We study proximity bounds within a natural model of random integer programs of the type $\max \boldsymbol{c}^{\top} \boldsymbol{x}: \boldsymbol{A x}=\boldsymbol{b}, \boldsymbol{x} \in \mathbb{Z}_{\geq 0}$, where $\boldsymbol{A} \in \mathbb{Z}^{m \times n}$ of rank $m, \boldsymbol{b} \in \mathbb{Z}^{m}$ and $\boldsymbol{c} \in \mathbb{Z}^{n}$. We prove that (up to a constant depending on $n$ ) the proximity is "generally" bounded by $\Delta_{m}(\boldsymbol{A})^{1 /(n-m)}$, where $\Delta_{m}(\boldsymbol{A})$ is the maximal absolute value of an $m \times m$ subdeterminant of $\boldsymbol{A}$. This is significantly better than the best deterministic bounds which are linear in $\Delta_{m}(\boldsymbol{A})$.
\end{abstract}

\section{Introduction}

Given an linear program of the form

$$
\begin{aligned}
\max \boldsymbol{c}^{\top} \boldsymbol{x}: \boldsymbol{A} \boldsymbol{x} & =\boldsymbol{b} \\
\boldsymbol{x} & \geq \mathbf{0},
\end{aligned}
$$

where $\boldsymbol{A}$ is a full-row-rank $m \times n$ integral matrix, $\boldsymbol{b} \in \mathbb{Z}^{m}$, and $\boldsymbol{c} \in \mathbb{Z}^{n}$, the proximity problem seeks to understand how far away an optimal feasible solution $\boldsymbol{x}^{*}$ can be to a nearby feasible integer solution $\boldsymbol{z}^{*}$. Assuming the feasible region has at least one such integral point, bounds for proximity are typically given in terms of the largest possible absolute value $\Delta_{m}(\boldsymbol{A})$ of any $m \times m$ subdeterminant of $\boldsymbol{A}$. This is a well-studied problem which goes back to the classic Cook et al. result 4 bounding the proximity of the dual of (1). See, for instance, the recent works of Eisenbrand and Weismantel [5] and of Aliev, Henk, and Oertel [1] and the references therein.

In this manuscript, we would like to understand the worst-possible proximity, which we denote by $\operatorname{dist}(\boldsymbol{A})$, over all choices of $\boldsymbol{b}$ and $\boldsymbol{c}$, when the matrix $\boldsymbol{A}$ is chosen randomly. The model of randomness we consider is the following: we choose the matrix $\boldsymbol{A}$ up to left-multiplication by unimodular matrices, and we choose

* The first author was funded by the Deutsche Forschungsgemeinschaft (DFG, German Research Foundation) under Germany's Excellence Strategy - The Berlin Mathematics Research Center MATH+(EXC-2046/1, project ID: 390685689). 
$\boldsymbol{A}$ uniformly at random subject to the condition that $\Delta(\boldsymbol{A}):=\sqrt{\operatorname{det} \boldsymbol{A} \boldsymbol{A}^{\top}}$ is at most some large, fixed integer $T$. This is a natural model to study from a geometric point of view, as $\Delta(\boldsymbol{A})$ is the determinant of the lattice of integer points in the kernel of $\boldsymbol{A}$. This is also the model considered by Aliev and Henk in [2], in their investigation of diagonal Frobenius numbers.

Our main result concerns not $\operatorname{dist}(\boldsymbol{A})$ but rather a related random variable we denote by $\operatorname{dist}^{*}(\boldsymbol{A})$. This is an asymptotic version of $\operatorname{dist}(\boldsymbol{A})$ that further imposes some mild restrictions on $\boldsymbol{b}$. Our main result is that it satisfies the following Markov-type inequality:

$$
\mathbf{P}\left(\operatorname{dist}^{*}(\boldsymbol{A})>t \Delta(\boldsymbol{A})^{1 /(n-m)}\right) \ll t^{-2 / 3} .
$$

Here $\ll$ means less than, up to constants which only depend on the dimension. In particular, this shows that proximity generally depends only on $\Delta^{1 /(n-m)}$ in the random setting, for "almost all" choices of $\boldsymbol{b}$ in a certain precise sense. This is significantly better than the linear dependency on $\Delta_{m}$ in the deterministic case, that is known to be tight [1, Theorem 1]. A similar result, with a slightly different random model, was obtained in [1] the so-called knapsack scenario, where $m=1$. We also mention recent work of Oertel, Paat, and Weismantel in 8, which considers a random model that allows $\boldsymbol{b}$ to vary but keeps $\boldsymbol{A}$ fixed.

The proof of this result combines ideas of [2] and [1] using facts from the geometry of numbers, some results of Schmidt from [9] on random sublattices of $\mathbb{Z}^{n}$ of fixed dimension, and computations of the measure of certain distinguished regions of the real Grassmannian $\operatorname{Gr}(n, d)$ of $d$-dimensional subspaces of $\mathbb{R}^{n}$. The idea is two-fold. First, we use the results of Schmidt to relate the discrete measure in our model to the continuous $O(n)$-invariant probability measure $\nu$ of $\operatorname{Gr}(n, d)$. We show that there are essentially two distinct "bad" regions of $\operatorname{Gr}(n, d)$, both parameterized by $t$, in which $\operatorname{dist}^{*}(\boldsymbol{A})$ could be large, but whose measure with respect to $\nu$ gets smaller as $t$ gets larger.

We remark that the exponent of $-2 / 3$ is mainly an artifact of the proof, and we expect that it can be further improved. The problem of finding an inequality analogous to $(2)$ for $\operatorname{dist}(\boldsymbol{A})$ is more challenging and remains open, as the polyhedral combinatorics of 1 may interfere with our analysis.

\section{Main result and notation}

\section{$2.1 \quad$ Notation}

Throughout this manuscript we assume fixed positive integers $d, m, n$ such that $n=m+d$. For a subset $\sigma \subseteq[n]$ and $\boldsymbol{x} \in \mathbb{R}^{n}$, we let $\boldsymbol{x}_{\sigma}$ denote the vector obtained by orthogonally projecting $\boldsymbol{x}$ onto the coordinates indexed by $\sigma$. Similarly, if $\boldsymbol{A}$ is a matrix, then we denote by $\boldsymbol{A}_{\sigma}$ the submatrix of $\boldsymbol{A}$ whose columns are those indexed by $\sigma$. In particular, if $k \in[n]$ then $\boldsymbol{A}_{k}$ denotes the corresponding column of $\boldsymbol{A}$. If $\boldsymbol{A}_{\sigma}$ is an invertible square matrix we say $\sigma$ is a basis of $\boldsymbol{A}$. We denote the complement of $\sigma$ by $\bar{\sigma}:=[n] \backslash \sigma$. Given a $d$-dimensional subspace $L \subseteq \mathbb{R}^{n}$, the $m$-dimensional orthogonal complement of $L$ is denoted by $L^{\perp}$. If $\Lambda \subset \mathbb{R}^{n}$, let $\Lambda_{\mathbb{R}}$ denote the linear subspace of $\mathbb{R}^{n}$ spanned by $\Lambda$. 


\subsection{Definition of $\operatorname{dist}(A)$}

Given a full-rank matrix $\boldsymbol{A} \in \mathbb{Z}^{m \times n}$ and vector $\boldsymbol{b} \in \mathbb{Z}^{m}$, we define the polyhedron

$$
\mathcal{P}(\boldsymbol{A}, \boldsymbol{b}):=\left\{\boldsymbol{x} \in \mathbb{R}^{n}: \boldsymbol{A x}=\boldsymbol{b}, \boldsymbol{x} \geq \mathbf{0}\right\} .
$$

Given a vertex $\boldsymbol{x}^{*}$ of this polyhedron, we define

$$
\operatorname{dist}\left(\boldsymbol{A}, \boldsymbol{b}, \boldsymbol{x}^{*}\right):=\inf _{\boldsymbol{z}^{*} \in \mathbb{Z}^{n} \cap \mathcal{P}(\boldsymbol{A}, \boldsymbol{b})}\left\|\boldsymbol{x}^{*}-\boldsymbol{z}^{*}\right\|_{2} .
$$

We then define the worst-case distance over all right-hand-side vectors $\boldsymbol{b} \in \mathbb{Z}^{m}$ for which $\mathcal{P}(\boldsymbol{A}, \boldsymbol{b})$ is nonempty, and over all vertices $\boldsymbol{x}^{*}$ of $\mathcal{P}(\boldsymbol{A}, \boldsymbol{b})$ :

$$
\operatorname{dist}(\boldsymbol{A}):=\sup _{\boldsymbol{b}} \sup _{\boldsymbol{x}^{*}} \operatorname{dist}\left(\boldsymbol{A}, \boldsymbol{b}, \boldsymbol{x}^{*}\right) .
$$

This definition has the disadvantage that it is stated in terms of the matrix $\boldsymbol{A}$. Since we may replace $\boldsymbol{A} \boldsymbol{x}=\boldsymbol{b}$ with $\boldsymbol{U} \boldsymbol{A} \boldsymbol{x}=\boldsymbol{U} \boldsymbol{b}$ for any unimodular $m \times m$ matrix $\boldsymbol{U}$, it is not so clear from this formulation how to define our random model. This motivates an alternative, more geometric definition of $\operatorname{dist}(\boldsymbol{A})$ which we now state.

\subsection{Definition of $\operatorname{dist}(\Lambda)$}

Suppose instead we start with a primitive $d$-dimensional sublattice $\Lambda$ of $\mathbb{Z}^{n}$, meaning that $\Lambda=\Lambda_{\mathbb{R}} \cap \mathbb{Z}^{n}$. Let $\boldsymbol{A} \in \mathbb{Z}^{m \times n}$ be any integral matrix for which the rows of $\boldsymbol{A}$ form a basis of $\Lambda_{\mathbb{R}}^{\perp} \cap \mathbb{Z}^{n}$. Suppose $\sigma$ is basis of $\boldsymbol{A}$, and $\boldsymbol{x}^{*}$ is a vector lying in the semigroup

$$
\mathcal{S}_{\sigma}:=\left\{\boldsymbol{x} \in \mathbb{Q}^{n}: \boldsymbol{x}_{\sigma} \in \boldsymbol{A}_{\sigma}^{-1} \mathbb{Z}^{m}, \boldsymbol{x}_{\bar{\sigma}}=\mathbf{0}, \boldsymbol{x}_{\sigma} \geq \mathbf{0}\right\}
$$

Then we may define

$$
\operatorname{dist}\left(\Lambda, \sigma, \boldsymbol{x}^{*}\right):=\operatorname{dist}\left(\boldsymbol{A}, \boldsymbol{b}, \boldsymbol{x}^{*}\right)
$$

where $\boldsymbol{b}:=\boldsymbol{A} \boldsymbol{x}^{*}$, and

$$
\operatorname{dist}(\Lambda):=\sup _{\sigma} \sup _{\boldsymbol{x}^{*}} \operatorname{dist}\left(\Lambda, \sigma, \boldsymbol{x}^{*}\right)
$$

where the supremum is taken over all bases $\sigma$ of $\boldsymbol{A}$ and elements $\boldsymbol{x}^{*} \in \mathcal{S}_{\sigma}$. Importantly, note that definitions (4) and (5) do not depent on the choice of $\boldsymbol{A}$, but only on the data $\left(\Lambda, \sigma, \boldsymbol{x}^{*}\right)$ and $\Lambda$, respectively.

One easily checks that the definitions of (3) and $\sqrt{5}$ agree. Nevertheless, the advantage of definition 5 is that there are only finitely many $d$-dimensional sublattices $\Lambda$ of $\mathbb{Z}^{n}$ whose determinant $\Delta(\Lambda):=\Delta(\boldsymbol{A})$ is at most some fixed positive integer $T$. Thus, we may consider the uniform distribution over these bounded-determinant lattices. 


\subsection{An asymptotic version of $\operatorname{dist}(\Lambda)$}

Again assume the rows of $\boldsymbol{A}$ form a basis of $\Lambda_{\mathbb{R}}^{\perp} \cap \mathbb{Z}^{n}$. We next consider a related version of $\operatorname{dist}(\boldsymbol{A})$, or equivalently $\operatorname{dist}(\Lambda)$. Let $B_{2}^{n} \subset \mathbb{R}^{n}$ denote the $n$ dimensional Euclidean ball. Define the vector $\boldsymbol{w} \in \mathbb{R}^{n}$ as follows: for each $i \in[n]$, set

$$
\boldsymbol{w}_{i}:=\sqrt{1-\boldsymbol{A}_{i}^{\top}\left(\boldsymbol{A} \boldsymbol{A}^{\top}\right)^{-1} \boldsymbol{A}_{i}} .
$$

This vector $\boldsymbol{w}$ measures, for each $i \in[n]$, the largest possible value of $\boldsymbol{x}_{i}$ for any $\boldsymbol{x} \in B_{2}^{n} \cap \Lambda_{\mathbb{R}}$. Denote by $\mu:=\mu\left(\Lambda, B_{2}^{n}\right)$ the covering radius of $B_{2}^{n}$ with respect to $A$. That is,

$$
\mu:=\inf \left\{t>0: \Lambda+t B_{2}^{n} \text { contains } \Lambda_{\mathbb{R}}\right\} .
$$

If $\sigma$ is a basis of $\boldsymbol{A}$ then define the following subsemigroup of $\mathcal{S}_{\sigma}$ :

$$
\mathcal{S}_{\sigma}^{*}:=\left\{\boldsymbol{x} \in \mathcal{S}_{\sigma}: \boldsymbol{x}_{\sigma} \geq \mu \boldsymbol{w}_{\sigma}+\boldsymbol{A}_{\sigma}^{-1} \boldsymbol{A}_{\bar{\sigma}} \boldsymbol{w}_{\bar{\sigma}}\right\} .
$$

The next proposition shows that if we further restrict $\boldsymbol{x}^{*}$ so that it can only lie in $\mathcal{S}_{\sigma}^{*}$, then we can guarantee that $\mathcal{P}(\boldsymbol{A}, \boldsymbol{b})$ contains an integral point reasonably close to $\boldsymbol{x}^{*}$. We prove it in Section 5

Proposition 1. For a basis $\sigma$ of $\boldsymbol{A}$ and $\boldsymbol{x}^{*} \in \mathcal{S}_{\sigma}^{*}$, let $\boldsymbol{b}=\boldsymbol{A} \boldsymbol{x}^{*}$. Then $\mathcal{P}(\boldsymbol{A}, \boldsymbol{b})$ contains a translate of the scaled ball $\mu \cdot\left(B_{2}^{n} \cap \Lambda_{\mathbb{R}}\right)$, which in turn contains an integral vector.

Now set

$$
\operatorname{dist}^{*}(\Lambda):=\sup _{\sigma} \sup _{\boldsymbol{x}^{*}} \operatorname{dist}\left(\Lambda, \sigma, \boldsymbol{x}^{*}\right),
$$

where the supremum is taken over all bases $\sigma$ of $\boldsymbol{A}$ and elements $\boldsymbol{x}^{*}$ of the semigroup $\mathcal{S}_{\sigma}^{*}$.

\subsection{Main result}

We are now ready to state the main theorem.

Theorem 1. For $T \gg 1$, let $\Lambda$ be a primitive sublattice of $\mathbb{Z}^{n}$ of dimension $d$ and determinant at most $T$, chosen uniformly at random. Then for all $t>1$,

$$
\mathbf{P}\left(\operatorname{dist}^{*}(\Lambda)>t(\Delta(\Lambda))^{1 / d}\right) \ll t^{-2 / 3}
$$

\section{A theorem of Schmidt}

In this section we state a result that is fundamental to the proof, which follows from the results of Schmidt in [9]. We continue with our assumption that $d=$ $n-m$. Let $\operatorname{Gr}(d, n)$ denote the set of $d$-dimensional subspaces of $\mathbb{R}^{n}$. Let $\nu$ denote the unique $O(n)$-invariant probability measure on the real Grassmannian $\operatorname{Gr}(d, n)$. 
Definition 1 ([9, p. 40]). A set $\xi \subset \operatorname{Gr}(d, n)$ is Jordan measurable if for all $\varepsilon>0$ there exists continuous functions $f_{1} \leq \mathbf{1}_{\xi} \leq f_{2}$ such that

$$
\int\left(f_{2}-f_{1}\right) d \nu<\varepsilon
$$

Here $\mathbf{1}_{\xi}$ denotes the indicator function of $\xi$.

Definition 2. Let $\boldsymbol{a}=\left(a_{1}, \ldots, a_{d}\right) \in \mathbb{R}^{d}$, with each $a_{i} \geq 1$. Let $T$ be a positive integer, and let $\xi \subset \operatorname{Gr}(d, n)$. Then we define $G(\boldsymbol{a}, \xi, T)$ to be the set of sublattices $\Lambda$ of $\mathbb{Z}^{n}$ of dimension $d$ with determinant at most $T$, such that

$$
\frac{\lambda_{i+1}(\Lambda)}{\lambda_{i}(\Lambda)} \geq a_{i} \text { for all } i=1,2, \ldots, d,
$$

and $\Lambda_{\mathbb{R}} \in \xi$.

The result of Schmidt that we intend to use is a combination of Theorems 3 and 5 in [9]:

Theorem 2. Assuming $\xi \subset \operatorname{Gr}(d, n)$ is Jordan measurable, we have

$$
|G(\boldsymbol{a}, \xi, T)| \asymp\left(\prod_{i=1}^{d-1} a_{i}^{-i(d-i)}\right) \nu(\xi) T^{n},
$$

where $f \asymp g$ means $f \ll g$ and $g \ll f$.

Let $G(d, n, T)$ denote the set of all sublattices of $\mathbb{Z}^{n}$ of dimension $d$ with determinant at most $T$. Let $\mathbf{P}=\mathbf{P}_{d, n, T}$ denote the uniform probability distribution over $G(d, n, T)$.

Corollary 1. For $t>1$, we have

$$
\mathbf{P}\left(\max _{i \in[d]}\left\{\frac{\lambda_{i+1}(\Lambda)}{\lambda_{i}(\Lambda)}\right\} \geq t\right) \ll(d-1) t^{-(d-1)} .
$$

Proof. Following Aliev and Henk in [2, let

$$
\boldsymbol{\delta}_{i}(t):=(1, \ldots, 1, t, 1, \ldots, 1)^{\top} \in \mathbb{R}^{d} .
$$

Applying the union bound to Theorem 2 this probability is at most

$$
\sum_{i=1}^{d-1} \frac{\left|G\left(\boldsymbol{\delta}_{i}(t), \operatorname{Gr}(d, n), T\right)\right|}{\left|G\left(\boldsymbol{\delta}_{i}(1), \operatorname{Gr}(d, n), T\right)\right|} \ll \sum_{i=1}^{d-1} t^{-i(d-i)} \leq(d-1) t^{-(d-1)} .
$$

\section{Typical Cramer's rule ratios}

This section is devoted to showing that the largest absolute value of any entry of the matrix $\boldsymbol{A}_{\sigma}^{-1} \boldsymbol{A}_{\bar{\sigma}}$ is typically not too large, when the subspace $L:=\operatorname{ker} \boldsymbol{A}$ is chosen uniformly at random from $\operatorname{Gr}(d, n)$. Note that the matrix $\boldsymbol{A}_{\sigma}^{-1} \boldsymbol{A}_{\bar{\sigma}}$ depends only on $L$ and $\sigma$. We remark that the entries of the matrix $\boldsymbol{A}_{\sigma}^{-1} \boldsymbol{A}_{\bar{\sigma}}$ are explicitly computed using Cramer's rule: for $i \in \sigma$ and $j \notin \sigma$, we have

$$
\left(\boldsymbol{A}_{\sigma}^{-1} \boldsymbol{A}_{j}\right)_{i}=\frac{\operatorname{det}\left(\boldsymbol{A}_{\sigma-i+j}\right)}{\operatorname{det}\left(\boldsymbol{A}_{\sigma}\right)} .
$$




\subsection{The real Grassmannian}

For a general introduction to matrix groups and Grassmannians, we refer the reader to 3. There is a right action of the orthogonal group $O(n)$ on $\operatorname{Gr}(d, n)$ defined as follows: if $\operatorname{ker}(\boldsymbol{A}) \in \operatorname{Gr}(d, n)$, where $\boldsymbol{A} \in \mathbb{R}^{m \times n}$, then

$$
(\operatorname{ker}(\boldsymbol{A})) \cdot \boldsymbol{U}=\operatorname{ker}(\boldsymbol{A} \boldsymbol{U}) \text {. }
$$

This is well-defined, since if $\operatorname{ker}(\boldsymbol{A})=\operatorname{ker}\left(\boldsymbol{A}^{\prime}\right)$ for some $\boldsymbol{A}^{\prime} \in \mathbb{R}^{m \times n}$, then $\boldsymbol{A}=D \boldsymbol{A}^{\prime}$ for some invertible $m \times m$ matrix $D$, and hence

$$
\operatorname{ker}(\boldsymbol{A} \boldsymbol{U})=\operatorname{ker}\left(D \boldsymbol{A}^{\prime} \boldsymbol{U}\right)=\operatorname{ker}\left(\boldsymbol{A}^{\prime} \boldsymbol{U}\right) .
$$

Let $\mathrm{St}^{m \times n}:=\left\{\boldsymbol{A} \in \mathbb{R}^{m \times n}: \operatorname{rank}(\boldsymbol{A})=m\right\}$. Call this the Stiefel manifold. Again, there is a right action of $O(n)$ on $\mathrm{St}^{m \times n}$ which in this case is simply right multiplication:

$$
\boldsymbol{A} \cdot \boldsymbol{U}=\boldsymbol{A} \boldsymbol{U} .
$$

The only thing to check here is that $\boldsymbol{A} \boldsymbol{U}$ indeed lies in $\mathrm{St}^{m \times n}$, but this is indeed the case since

$$
\boldsymbol{A} \boldsymbol{U}(\boldsymbol{A U})^{\top}=\boldsymbol{A} \boldsymbol{U} \boldsymbol{U}^{\top} \boldsymbol{A}^{\top}=\boldsymbol{A} \boldsymbol{A}^{\top},
$$

thus $\boldsymbol{A}$ and $\boldsymbol{A} \boldsymbol{U}$ have the same Gram matrix $\boldsymbol{A} \boldsymbol{A}^{\top}$, and a $m \times n$ matrix has full-rank if and only if its Gram matrix does.

The kernel map gives rise to a surjective map

$$
\begin{aligned}
\operatorname{ker}: \mathrm{St}^{m \times n} & \rightarrow \operatorname{Gr}(d, n) \\
\boldsymbol{A} & \mapsto \operatorname{ker}(\boldsymbol{A})
\end{aligned}
$$

Thus, we see from (8) that the following statement holds:

Proposition 2. The map ker : $\mathrm{St}^{m \times n} \rightarrow \operatorname{Gr}(d, n)$ is equivariant with respect to the right actions of $O(n)$ on $\mathrm{St}^{m \times n}$ and $\operatorname{Gr}(d, n)$; that is, $(\operatorname{ker}(\boldsymbol{A})) \cdot \boldsymbol{U}=$ $\operatorname{ker}(\boldsymbol{A} \cdot \boldsymbol{U})$.

\subsection{Probability spaces}

Consider the probability space $\left(\mathbb{R}^{m \times n}, \mathscr{B}\left(\mathbb{R}^{m \times n}\right), \gamma\right)$ where $\mathscr{B}\left(\mathbb{R}^{m \times n}\right)$ is the Borel $\sigma$-algebra, and the measure $\gamma$ is defined so that each $\boldsymbol{A} \in \mathbb{R}^{m \times n}$ has iid $N(0,1)$ entries. In other words, $\gamma$ is the standard Gaussian probability measure on the $m n$-dimensional real vector space $\mathbb{R}^{m \times n}$ with mean zero and identity covariance matrix. By restricting to $\mathrm{St}^{m \times n}$, we get the probability space $\left(\mathrm{St}^{m \times n}, \mathscr{B}\left(\mathrm{St}^{m \times n}\right), \gamma\right)$. We can do this because $\mathbb{R}^{m \times n} \backslash \mathrm{St}^{m \times n}$ is an algebraic hypersurface in $\mathbb{R}^{m \times n}$, and therefore has measure zero with respect to $\gamma$. Let $\mathscr{B}:=\mathscr{B}\left(\mathrm{St}^{m \times n}\right)$.

The Grassmannian $\operatorname{Gr}(d, n)$ is endowed with the topology where $E \subseteq \operatorname{Gr}(d, n)$ is open iff $\operatorname{ker}^{-1}(E)$ is open in $\mathrm{St}^{m \times n}$. Let $\mathscr{G}$ denote the associated Borel $\sigma$ algebra. As before, we let $\nu: \mathscr{G} \rightarrow[0,1]$ denote the $O(n)$-invariant probability measure on $\operatorname{Gr}(d, n)$. This measure is characterized as follows: 
Proposition 3 ([7, Corollary 3.1.3]). The measure $\nu$ is the unique measure on $\operatorname{Gr}(d, n)$ satisfying

$$
\begin{aligned}
\nu(E \cdot \boldsymbol{U}) & =\nu(E) \text { for all } E \in \mathscr{G} \text { and } \boldsymbol{U} \in O(n) \\
\nu(\operatorname{Gr}(d, n)) & =1 .
\end{aligned}
$$

The map ker $: \mathrm{St}^{m \times n} \rightarrow \operatorname{Gr}(d, n)$ thus defines a map of probability spaces:

$$
\text { ker }:\left(\mathrm{St}^{m \times n}, \mathscr{B}, \gamma\right) \rightarrow(\operatorname{Gr}(d, n), \mathscr{G}, \nu) \text {. }
$$

Proposition 4. The measure $\nu$ is the pushforward measure of $\gamma$ under this map. That is, $\nu(E)=\gamma\left(\operatorname{ker}^{-1}(E)\right)$ for each $E \in \mathscr{G}$.

Proof. We establish the conditions of (9). By surjectivity, and the fact that $\gamma$ is a probability measure, we have

$$
\gamma\left(\operatorname{ker}^{-1}(\operatorname{Gr}(d, n))\right)=\gamma\left(\mathrm{St}^{m \times n}\right)=1 .
$$

It therefore remains to show $\gamma\left(\operatorname{ker}^{-1}(E \cdot \boldsymbol{U})\right)=\gamma\left(\operatorname{ker}^{-1}(E)\right)$ for each $E \in \mathscr{G}$ and $\boldsymbol{U} \in O(n)$. By Proposition 2, we have

$$
\operatorname{ker}^{-1}(E \cdot \boldsymbol{U})=\operatorname{ker}^{-1}(E) \cdot \boldsymbol{U}
$$

Now, $\mathbb{R}^{m \times n}$ has the inner product $\langle\boldsymbol{A}, \boldsymbol{B}\rangle=\operatorname{trace}\left(\boldsymbol{A} \boldsymbol{B}^{\top}\right)$. With respect to this inner product we may consider the subgroup $O(m \times n)$ of GL $\left(\mathbb{R}^{m \times n}\right)$ which is given by

$$
O(m \times n):=\left\{\varphi \in \mathrm{GL}\left(\mathbb{R}^{m \times n}\right):\langle\varphi(\boldsymbol{A}), \varphi(\boldsymbol{B})\rangle=\langle\boldsymbol{A}, \boldsymbol{B}\rangle\right\} .
$$

Observe that, for a fixed $\boldsymbol{U} \in O(n)$, the linear map $\varphi_{\boldsymbol{U}} \in \mathrm{GL}\left(\mathbb{R}^{m \times n}\right)$ given by

$$
\varphi_{U}(\boldsymbol{A})=\boldsymbol{A U}
$$

lies in $O(m \times n)$, since

$$
\langle\varphi(\boldsymbol{A}), \varphi(\boldsymbol{B})\rangle=\operatorname{trace}\left(\boldsymbol{A U}(\boldsymbol{B} \boldsymbol{U})^{\top}\right)=\operatorname{trace}\left(\boldsymbol{A} \boldsymbol{B}^{\top}\right)=\langle\boldsymbol{A}, \boldsymbol{B}\rangle
$$

Now the probability measure $\gamma$ on $\mathbb{R}^{m \times n}$ is defined so that the coordinates $\boldsymbol{A}_{i, j}$ of a randomly chosen $\boldsymbol{A} \in \mathbb{R}^{m \times n}$ are iid $N(0,1)$ normally distributed. In particular this measure is invariant under isometry, in that for all $\mathcal{K} \in \mathscr{B}\left(\mathbb{R}^{m \times n}\right)$ and $\varphi \in O(m \times n)$, we have

$$
\gamma(\varphi(\mathcal{K}))=\gamma(\mathcal{K})
$$

The same is therefore true for the restricted probability measure $\gamma$ on $\mathrm{St}^{m \times n}$. It follows that if $\boldsymbol{U} \in O(n)$ and $E \in \mathscr{G}$, then, using (10), [11, and [12, we have

$$
\gamma\left(\operatorname{ker}^{-1}(E \cdot \boldsymbol{U})\right)=\gamma\left(\operatorname{ker}^{-1}(E) \cdot \boldsymbol{U}\right)=\gamma\left(\varphi_{\boldsymbol{U}}\left(\operatorname{ker}^{-1}(E)\right)\right)=\gamma\left(\operatorname{ker}^{-1}(E)\right) .
$$




\subsection{Cramer's rule ratios}

Let $\sigma \subset[n]$ of size $m$, and define

$$
\begin{aligned}
\mathrm{St}_{\sigma}^{m \times n} & :=\left\{\boldsymbol{A} \in \mathrm{St}^{m \times n}: \boldsymbol{A}_{\sigma} \text { is nonsingular }\right\} . \\
\operatorname{Gr}(d, n)_{\sigma} & :=\left\{\operatorname{ker}(\boldsymbol{A}) \in \operatorname{Gr}(d, n): \boldsymbol{A}_{\sigma} \text { is nonsingular }\right\} .
\end{aligned}
$$

Note that $\gamma\left(\operatorname{St}_{\sigma}^{m \times n}\right)=\nu\left(\operatorname{Gr}(n, d)_{\sigma}\right)=1$. Also define, for $s>1, i \in \sigma$, and $j \notin \sigma$,

$$
\xi_{\sigma, i, j}(s):=\left\{\operatorname{ker}(\boldsymbol{A}) \in \operatorname{Gr}(d, n)_{\sigma}:\left|\left(\boldsymbol{A}_{\sigma}^{-1} \boldsymbol{A}_{j}\right)_{i}\right|>s\right\} .
$$

Proposition 5. For $s>1$ and $\sigma, i, j$ as above, we have

$$
\nu\left(\xi_{\sigma, i, j}(s)\right)=\frac{2}{\pi s}+\mathcal{O}\left(s^{-3}\right) .
$$

Proof. Let $\boldsymbol{A}$ be a random element of $\mathrm{St}_{\sigma}^{m \times n}$, and let $H$ denote the (random) hyperplane spanned by the columns of $\boldsymbol{A}_{\sigma-i}$, and let $\ell$ denote the line perpendicular to $H$. Let $\boldsymbol{u}_{\ell}$ denote the unit normal vector to $H$ whose first nonzero coordinate is positive. Thus,

$$
\ell=\mathbb{R} \boldsymbol{u}_{\ell}=\left\{\lambda \boldsymbol{u}_{\ell}: \lambda \in \mathbb{R}\right\} .
$$

Let $\alpha \in\{-1,+1\}$ denote the sign of the first nonzero entry of $\mathbf{e}_{i}^{\top} \boldsymbol{A}_{\sigma}^{-1}$. Then we can write

$$
\boldsymbol{u}_{\ell}^{\top}=\frac{\alpha \mathbf{e}_{i}^{\top} \boldsymbol{A}_{\sigma}^{-1}}{\left\|\mathbf{e}_{i}^{\top} \boldsymbol{A}_{\sigma}^{-1}\right\|_{2}},
$$

since for all $k \in \sigma-i$ we have

$$
\alpha \mathbf{e}_{i}^{\top} \boldsymbol{A}_{\sigma}^{-1} \boldsymbol{A}_{k}=\alpha \mathbf{e}_{i}^{\top} \boldsymbol{A}_{\sigma}^{-1} \boldsymbol{A}_{\sigma} \mathbf{e}_{k}=0
$$

and $\alpha \mathbf{e}_{i}^{\top} \boldsymbol{A}_{\sigma}^{-1}$ has first nonzero component positive by definition of $\alpha$.

Now let $k$ be any element of $[n]$ outside of $\sigma-i$. Since $\boldsymbol{u}_{\ell}$ depends only on $\boldsymbol{A}_{\sigma-i}$, and the entries of $\boldsymbol{A}$ are mutually independent, we have that $\boldsymbol{u}_{\ell}$ and $\boldsymbol{A}_{k}$ are independent random vectors. Now, for any fixed unit vector $\boldsymbol{v} \in \mathbb{S}^{n-1}$, as $\boldsymbol{A}_{k}$ has $N(0,1)$ iid entries, then the dot product $\boldsymbol{v}^{\top} \boldsymbol{A}_{k}$ also has distribution $N(0,1)$. Thus, for any fixed $t \in \mathbb{R}$, the random variable

$$
\gamma\left(\boldsymbol{u}_{\ell}^{\top} \boldsymbol{A}_{k} \leq t \mid \ell\right)
$$

(i.e. the conditional probability in terms of the $\sigma$-algebra generated by $\ell$ ) is in fact constant. Evaluating at the line $\ell=\mathbb{R} \mathbf{e}_{1}$, for example, this constant is given by

$$
\gamma\left(\boldsymbol{A}_{1, k} \leq t\right) .
$$

This shows that the random quantity $\boldsymbol{u}_{\ell}^{\top} \boldsymbol{A}_{k}$ has distribution $N(0,1)$. We have

$$
\left(\boldsymbol{A}_{\sigma}^{-1} \boldsymbol{A}_{j}\right)_{i}=\frac{\mathbf{e}_{i}^{\top} \boldsymbol{A}_{\sigma}^{-1} \boldsymbol{A}_{j}}{\mathbf{e}_{i}^{\top} \boldsymbol{A}_{\sigma}^{-1} \boldsymbol{A}_{i}}=\frac{\boldsymbol{u}_{\ell}^{\top} \boldsymbol{A}_{j}}{\boldsymbol{u}_{\ell}^{\top} \boldsymbol{A}_{i}}
$$


The independence of $\boldsymbol{u}_{\ell}^{\top} \boldsymbol{A}_{i}$ and $\boldsymbol{u}_{\ell}^{\top} \boldsymbol{A}_{j}$ imply that $\left(\boldsymbol{A}_{\sigma}^{-1} \boldsymbol{A}_{j}\right)_{i}$ has the Cauchy distribution, that is, the ratio of two iid $N(0,1)$ random variables. In particular, the cdf of $\left(\boldsymbol{A}_{\sigma}^{-1} \boldsymbol{A}_{j}\right)_{i}$ is given by

$$
\gamma\left(\left(\boldsymbol{A}_{\sigma}^{-1} \boldsymbol{A}_{j}\right)_{i} \leq t\right)=\frac{1}{\pi} \arctan (t)+\frac{1}{2} .
$$

See [6, p. 50] for more on the Cauchy distribution. Using the series expansion

$$
\arctan (t)=\frac{\pi}{2}-\frac{1}{t}+\frac{1}{3 t^{3}}-\frac{1}{5 t^{5}}+\cdots,
$$

we get

$$
\gamma\left(\left(\boldsymbol{A}_{\sigma}^{-1} \boldsymbol{A}_{j}\right)_{i} \leq t\right)=1-\left(\frac{1}{\pi t}-\frac{1}{3 \pi t^{3}}+\frac{1}{5 \pi t^{5}}-\cdots\right) .
$$

Hence, using Proposition 4 and the fact $s>1$, we conclude

$$
\begin{aligned}
\nu\left(\xi_{\sigma, i, j}(s)\right) & =\gamma\left(\left|\left(\boldsymbol{A}_{\sigma}^{-1} \boldsymbol{A}_{j}\right)_{i}\right|>s\right) \\
& =2 \cdot \gamma\left(\left(\boldsymbol{A}_{\sigma}^{-1} \boldsymbol{A}_{j}\right)_{i}>s\right) \\
& =2\left(1-\gamma\left(\left(\boldsymbol{A}_{\sigma}^{-1} \boldsymbol{A}_{j}\right)_{i} \leq s\right)\right) \\
& =2\left(\frac{1}{\pi s}-\frac{1}{3 \pi s^{3}}+\frac{1}{5 \pi s^{5}}-\cdots\right) \\
& =\frac{2}{\pi s}+\mathcal{O}\left(s^{-3}\right) .
\end{aligned}
$$

\section{Proof of main result}

In this final section we prove the main result of this paper, Theorem 1

Definition 3. Define the constant

$$
\tilde{\omega}_{d}:=\frac{\omega_{d}^{1 / d}}{d},
$$

where $\omega_{d}$ denotes the volume of the d-dimensional Euclidean ball. This constant $\tilde{\omega}_{d}$ is of the order $d^{-3 / 2}$.

Definition 4. Assume $\Lambda_{\mathbb{R}}=\operatorname{ker}(\boldsymbol{A})$. Given positive real numbers $s$ and $u$, we say $\Lambda$ is $(\sigma, s, u)$-controlled if $\sigma$ is a basis of $\boldsymbol{A}$ and:

1. The largest entry of $\boldsymbol{A}_{\sigma}^{-1} \boldsymbol{A}_{\bar{\sigma}}$ is at most s, and

2. The successive minima ratios of $\Lambda$ are not too large: we have

$$
\frac{\lambda_{i+1}(\Lambda)}{\lambda_{i}(\Lambda)}<\left(\tilde{\omega}_{d} u\right)^{2 /(d-1)}
$$

for all $i=1,2, \ldots, d-1$. 
Lemma 1 ([2, Proof of Lemma 5.2]). Suppose that

$$
\frac{\lambda_{i+1}(\Lambda)}{\lambda_{i}(\Lambda)}<\left(\tilde{\omega}_{d} u\right)^{2 /(d-1)}
$$

for all $i=1,2, \ldots, d-1$. Then

$$
\mu<u(\Delta(\Lambda))^{1 / d}
$$

Lemma 2. If $\sigma$ is a basis of $\boldsymbol{A}$ and $\Lambda$ is $(\sigma, s, u)$-controlled, then for all $\boldsymbol{x}^{*} \in \mathcal{S}_{\sigma}$ we have

$$
\operatorname{dist}\left(\Lambda, \sigma, \boldsymbol{x}^{*}\right) \leq 2 n^{3 / 2} \operatorname{su}(\Delta(\Lambda))^{1 / d} .
$$

Proof. Let $\boldsymbol{b}=\boldsymbol{A} \boldsymbol{x}^{*}$, let $B=B_{2}^{n} \cap \Lambda_{\mathbb{R}}$, and let $\mu$ denote the covering radius of $B$ with respect to $\Lambda$. Define the vector $\boldsymbol{v} \in \mathbb{R}^{n}$ so that:

$$
\begin{aligned}
\boldsymbol{v}_{j} & =\mu \boldsymbol{w}_{j} \text { for all } j \in \bar{\sigma} \\
\boldsymbol{A} \boldsymbol{v} & =\boldsymbol{b} .
\end{aligned}
$$

We show that the scaled, translated ball $\mu B+\boldsymbol{v}$ is contained in $\mathcal{P}(\boldsymbol{A}, \boldsymbol{b})$. Since $B \subseteq \Lambda_{\mathbb{R}}$, we have that each $\boldsymbol{x} \in \mu B+\boldsymbol{v}$ satisfies $\boldsymbol{A} \boldsymbol{x}=\boldsymbol{b}$. For each $j \in[n]$, let $\boldsymbol{x}^{(j)}$ be the unique point in $\mu B+\boldsymbol{v}$ such that $\boldsymbol{x}_{j}^{(j)}$ is minimized. If $j \in \bar{\sigma}$, then

$$
\boldsymbol{x}_{j}^{(j)}=\mu\left(-\boldsymbol{w}_{j}\right)+\boldsymbol{v}_{j}=\mu\left(-\boldsymbol{w}_{j}\right)+\mu \boldsymbol{w}_{j}=0 .
$$

If $j \in \sigma$, then since $\boldsymbol{x}^{*} \in \mathcal{S}_{\sigma}$ we have

$$
\begin{aligned}
\boldsymbol{x}_{j}^{(j)} & =\mu\left(-\boldsymbol{w}_{j}\right)+\boldsymbol{v}_{j} \\
& =\mu\left(-\boldsymbol{w}_{j}\right)+\left(\boldsymbol{A}_{\sigma}^{-1} \boldsymbol{b}-\boldsymbol{A}_{\sigma}^{-1} \boldsymbol{A}_{\bar{\sigma}} \boldsymbol{w}_{\bar{\sigma}}\right)_{j} \\
& \geq \mu\left(-\boldsymbol{w}_{j}\right)+\mu \boldsymbol{w}_{j} \\
& =0 .
\end{aligned}
$$

This concludes the proof that $\mu B+\boldsymbol{v} \subseteq \mathcal{P}(\boldsymbol{A}, \boldsymbol{b})$.

Now, since $\mu$ is the covering radius of $B$ with respect to $\Lambda$, there exists $\boldsymbol{z}^{*} \in \mathbb{Z}^{n} \cap(\mu B+\boldsymbol{v})$ such that

$$
\left\|\boldsymbol{x}^{*}-\boldsymbol{z}^{*}\right\|_{2} \leq\left\|\boldsymbol{x}^{*}-\boldsymbol{v}\right\|_{2}+\left\|\boldsymbol{v}-\boldsymbol{z}^{*}\right\|_{2} \leq \mu\|\tilde{\boldsymbol{w}}\|_{2}+\mu .
$$

where we define $\tilde{\boldsymbol{w}}:=\left(\boldsymbol{v}-\boldsymbol{x}^{*}\right) / \mu$. That is, $\tilde{\boldsymbol{w}}$ satisfies

$$
\begin{aligned}
\boldsymbol{A} \tilde{\boldsymbol{w}} & =\mathbf{0} \\
\tilde{\boldsymbol{w}} & =\boldsymbol{w}_{j} \text { for all } j \in \bar{\sigma} .
\end{aligned}
$$

Observe that

$$
\tilde{\boldsymbol{w}}_{\sigma}=-\boldsymbol{A}_{\sigma}^{-1} \boldsymbol{A}_{\bar{\sigma}} \tilde{\boldsymbol{w}}_{\bar{\sigma}}
$$


Using the fact $\boldsymbol{w} \in[0,1]^{n}$, we therefore have

$$
\begin{aligned}
\|\tilde{\boldsymbol{w}}\|_{2}^{2} & =\left\|\tilde{\boldsymbol{w}}_{\sigma}\right\|_{2}^{2}+\left\|\tilde{\boldsymbol{w}}_{\bar{\sigma}}\right\|_{2}^{2} \\
& =\left\|\boldsymbol{A}_{\sigma}^{-1} \boldsymbol{A}_{\bar{\sigma}} \tilde{\boldsymbol{w}}_{\bar{\sigma}}\right\|_{2}^{2}+\left\|\tilde{\boldsymbol{w}}_{\bar{\sigma}}\right\|_{2}^{2} \\
& \leq m\left\|\boldsymbol{A}_{\sigma}^{-1} \boldsymbol{A}_{\bar{\sigma}}\right\|_{\infty}^{2}\left\|\tilde{\boldsymbol{w}}_{\bar{\sigma}}\right\|_{1}^{2}+\left\|\tilde{\boldsymbol{w}}_{\bar{\sigma}}\right\|_{2}^{2} \\
& \leq\left(m s^{2}+1\right) d^{2} .
\end{aligned}
$$

Thus we conclude

$$
\begin{aligned}
\left\|\boldsymbol{x}^{*}-\boldsymbol{z}^{*}\right\|_{2} & \leq \mu\left(\|\tilde{\boldsymbol{w}}\|_{2}+1\right) \\
& \leq u \Delta^{1 / d}\left(\sqrt{\left(m s^{2}+1\right) d^{2}}+1\right) \\
& \leq 2 n^{3 / 2} s u \Delta^{1 / d} .
\end{aligned}
$$

Proof (Proof of Theorem 1). Let $\Lambda$ be a uniformly chosen lattice from $G(d, n, T)$. Let $t>1$, and let $s:=t^{2 / 3} /\left(2 n^{3 / 2}\right)$ and $u:=t^{1 / 3}$, so that $t=2 n^{3 / 2} s u$ as in Lemma 2. We have

$$
\begin{aligned}
& \mathbf{P}\left(\operatorname{dist}(\Lambda)>t(\Delta(\Lambda))^{1 / d}\right) \\
& \leq \sum_{\sigma} \mathbf{P}\left(\sigma \text { basis of } \boldsymbol{A}, \operatorname{dist}\left(\Lambda, \sigma, \boldsymbol{x}^{*}\right)>t(\Delta(\Lambda))^{1 / d} \text { for some } \boldsymbol{x}^{*} \in \mathcal{S}_{\sigma}\right) \\
& \leq \sum_{\sigma} \mathbf{P}(\sigma \text { basis of } \boldsymbol{A}, \Lambda \text { is not }(\sigma, s, u) \text {-controlled })
\end{aligned}
$$

where the sums are over all subsets $\sigma \subseteq[n]$ of size $m$. It therefore suffices to show, for each such $\sigma$,

$$
\mathbf{P}(\sigma \text { basis of } \boldsymbol{A}, \Lambda \text { is not }(\sigma, s, u) \text {-controlled }) \ll t^{-2 / 3} \text {. }
$$

By definition, this probability is at most

$$
\mathbf{P}\left(\max _{i \in[d]}\left\{\frac{\lambda_{i+1}(\Lambda)}{\lambda_{i}(\Lambda)}\right\} \geq\left(\tilde{\omega}_{d} u\right)^{2 /(d-1)}\right)+\sum_{\substack{i \in \sigma \\ j \notin \sigma}} \mathbf{P}\left(\sigma \text { basis of } \boldsymbol{A}, \quad\left(\boldsymbol{A}_{\sigma}^{-1} \boldsymbol{A}_{j}\right)_{i} \geq s\right) .
$$

By Theorem 2, we have

$$
\mathbf{P}\left(\sigma \text { basis of } \boldsymbol{A}, \quad\left(\boldsymbol{A}_{\sigma}^{-1} \boldsymbol{A}_{j}\right)_{i} \geq s\right)=\frac{\left|G\left(\mathbf{1}, \xi_{\sigma, i, j}(s), T\right)\right|}{|G(\mathbf{1}, \operatorname{Gr}(d, n), T)|} \asymp \nu\left(\xi_{\sigma, i, j}(s)\right) .
$$

Hence, applying Corollary 1 and Proposition 5 for $T$ sufficiently large, we may estimate up to constants the quantity 14 by

$$
u^{-2}+s^{-1} \ll t^{-2 / 3} \text {. }
$$




\section{References}

1. I. Aliev, M. Henk, and T. Oertel. Distances to lattice points in knapsack polyhedra. Math. Program., 182(1-2, Ser. A):175-198, 2020.

2. Iskander Aliev and Martin Henk. Feasibility of integer knapsacks. SIAM Journal on Optimization, 20(6):2978-2993, 2010.

3. A. Baker. Matrix Groups: An Introduction to Lie Group Theory. Springer Undergraduate Mathematics Series. Springer London, 2003.

4. W. Cook, A. M. H. Gerards, A. Schrijver, and É. Tardos. Sensitivity theorems in integer linear programming. Mathematical Programming, 34(3):251-264, Apr 1986.

5. Friedrich Eisenbrand and Robert Weismantel. Proximity results and faster algorithms for integer programming using the Steinitz lemma. ACM Trans. Algorithms, 16(1), November 2019.

6. V. Feller and W. Feller. An Introduction to Probability Theory and Its Applications, Volume 1. A Wiley publication in mathematical statistics. Wiley, 1968.

7. S.G. Krantz and H.R. Parks. Geometric Integration Theory. Cornerstones. Birkhäuser Boston, 2008.

8. Timm Oertel, Joseph Paat, and Robert Weismantel. The distributions of functions related to parametric integer optimization. SIAM Journal on Applied Algebra and Geometry, 4(3):422-440, 2020.

9. Wolfgang M Schmidt. The distribution of sublattices of $\mathbf{Z}^{m}$. Monatshefte für Mathematik, 125(1):37-81, 1998. 